\title{
ARTICLE
}

Received 6 Apr 2015 | Accepted 25 Jun 2015 | Published 3 Aug 2015 DOl: 10.1038/ncomms8923 OPEN

\section{Large-volume en-bloc staining for electron microscopy-based connectomics}

Yunfeng Hua ${ }^{1}$, Philip Laserstein ${ }^{1} \&$ Moritz Helmstaedter ${ }^{1}$

Large-scale connectomics requires dense staining of neuronal tissue blocks for electron microscopy (EM). Here we report a large-volume dense en-bloc EM staining protocol that overcomes the staining gradients, which so far substantially limited the reconstructable volumes in three-dimensional (3D) EM. Our protocol provides densely reconstructable tissue blocks from mouse neocortex sized at least $1 \mathrm{~mm}$ in diameter. By relaxing the constraints on precise topographic sample targeting, it makes the correlated functional and structural analysis of neuronal circuits realistic.

\footnotetext{
${ }^{1}$ Department of Connectomics, Max Planck Institute of Brain Research, Max-von-Laue-Strasse 4, D-60438 Frankfurt, Germany. Correspondence and requests for materials should be addressed to Y.H. (email: yunfeng.hua@brain.mpg.de) or to M.H. (email: mh@brain.mpg.de).
} 
M apping neuronal circuits densely is the goal of connectomics. Current imaging methods in highresolution connectomics require en-bloc staining of neuronal tissue for large-volume $\mathrm{EM}^{1}$. Establishing high-contrast staining in every neurite and synapse in large volumes homogeneously is, however, a substantial challenge to the employed staining protocols. In spite of research into the chemistry of heavy metal deposition in biological samples over several decades, the limitation of the employed protocols to small-dimension samples (typically $\sim 100-200-\mu$ m maximum penetration depth) had been widely accepted. Only very recently, a protocol aimed at whole-brain staining has been proposed ${ }^{2}$, which, however, is focused on very large whole-brain-sized samples and involves staining procedures in the scale of months, not days. We developed a staining protocol that overcomes the limitations of sample size with a simple, fast and reliable process, which provides high-contrast full-neuropil staining for large (millimetre size) volumes.

\section{Results}

The widely employed existing EM staining protocols either are limited to small volumes, yielding prohibitive staining gradients for larger structures (Fig. 1a-c), or stain only a subset of the neurites (myelinated fibre staining, Fig. 1d), with the additional requirement for charging compensation ${ }^{2}$. Our protocol provides, however, high-contrast full-neuropil staining for large (millimetre size) volumes (Fig. 1e). Its chemical logic, key steps and usability for dense circuit reconstruction are described in the following.

Neuronal tissue staining for high-resolution connectomics requires the deposition of heavy metal compounds into the membranes that outline neuronal cell bodies, their dendrites, axons and synaptic vesicles. Osmium (Os) and uranium compounds have been the most widely employed membrane contrasting agents for decades ${ }^{4-6}$, and uranyl acetate (UA) has also been implied in labelling proteins such as those in the postsynaptic density ${ }^{5}$. Alterations to both the Os tetroxide $\left(\mathrm{OsO}_{4}\right)$ and UA impregnation steps are at the core of our protocol.

To achieve high-contrast staining throughout large tissue blocks, the relevant compounds have to diffuse from the exterior through many successive lipid membranes to the centre of the tissue block. Secondly, these compounds have to react with the target structures in the membranes (or be deposited in the membrane by other means). There is a tradeoff between these two goals, since processes that enhance membrane attachment will reduce the availability of compounds for further diffusion, and vice versa. This is especially true when enhancing reactions are employed that further increase compound attachment, but thus decrease diffusability. One important enhancing reaction is the application of ferrocyanide for enhanced Os staining, which will be discussed below.

In brief, our protocol alterations therefore centre around improving the optimum between tissue penetration and membrane contrast. By separating the $\mathrm{OsO}_{4}$ penetration from the ferrocyanide enhancement reaction, by leaving out the intermittent washing step and by using a two-step temperature protocol in the UA impregnation, we achieve our homogeneous high-contrast penetration results as shown in Fig. 1e. Most notably, our protocol completely abolishes the intense staining ring generated in the state-of-the-art en-bloc staining protocols (Fig. 1b,c) which had been implied in blocking further stain penetration. When imaged at high resolution (Fig. 2), we find that samples stained with our protocol show intense and continuous membrane contrast (Fig. 2e,f and Supplementary Fig. 1) at about $500 \mu \mathrm{m}$ from the sample surface (Fig. 2a) with no indication of contrast decrease over this penetration distance.
Our protocol alterations are based on three notions about the underlying diffusion and reaction steps. We report these notions here to illustrate the reasoning that guided our protocol development (Fig. 3). First, we assumed that the classic $\mathrm{OsO}_{4}$ alkene reaction mechanism (the Sharpless dihydroxylation reaction ${ }^{7-9}$ ) is at the core of the $\mathrm{OsO}_{4}-$ membrane interactions (Fig. 3a). Second, we find indications that in addition to the covalent binding of $\mathrm{OsO}_{4}$ to unsaturated lipids, quadruply reduced $\mathrm{Os}^{\mathrm{IV}}$, made available as $\mathrm{OsO}_{2}$ by dismutation of $\mathrm{Os}^{\mathrm{VI}}$ compounds (Fig. 3b), and dissolved in the membrane, substantially contributes to membrane contrast. Finally, we exploit the fact that the diffusability and reactivity of UA is differentially affected by temperature, such that lower temperature reduces reactivity ${ }^{10}$ more strongly than it reduces diffusibility.

These reaction mechanisms imply that $\mathrm{OsO}_{4}$ diffuses across membranes by switching between its ionized hydrophilic form $\left[\mathrm{OsO}_{4}(\mathrm{OH})_{2}\right]^{2-}$ and its non-polar lipophilic form $\mathrm{OsO}_{4}$ (Fig. 3a, step 1 when entering the membrane, inverted step 1 (' -1 ') when exiting the membrane, see sketch in Fig. 3c). After entering the membrane, $\mathrm{OsO}_{4}$ can form cyclic osmate esters by covalent binding to unsaturated membrane lipids ${ }^{11-13}$ (Fig. 3a, step 2). However, these intermediates slowly undergo dihydroxylation $^{11,14}$ (Fig. 3a, step 3), with the effect of Os leaving the membrane again, yielding $\left[\mathrm{OsO}_{2}(\mathrm{OH})_{4}\right]^{2-}$, a water soluble Os compound at oxidation level VI. This step alone would result in membrane de-staining; however, it has been suggested based on chemical analysis ${ }^{12}$ that instable $\mathrm{Os}^{\mathrm{VI}}$ compounds dismutate quickly to $\mathrm{OsO}_{4}$ and $\mathrm{OsO}_{2}$ (Fig. 3b, step 5). $\mathrm{OsO}_{2}$ is non-polar and thus likely lipophilic and will be dissolved in the membrane, thus securing in-membrane Os deposition.

Following this logic, the main effect of reducing agents such as potassium ferrocyanide $\left(\mathrm{K}_{4} \mathrm{Fe}(\mathrm{CN})_{6}\right.$, step 4 in Fig. 3a) is to convert the VIII-oxidized water-soluble Os form into a VI-oxidized water-soluble form ${ }^{15}$, which makes more $\mathrm{Os}^{\mathrm{VI}}$ available for the dismutation step (Fig. 3b, step 5), and thus generates additional $\mathrm{OsO}_{2}$ to be deposited in the membrane. This pathway circumvents the osmate ester formation step (Fig. 3a, step 2), which requires unsaturated membrane lipids as reaction partners ${ }^{15}$.

Together, this would imply that the availability of Os ${ }^{\text {VIII }}$ for sequential membrane uptake/loss events (steps $1,-1$ ) is essential for the penetration of membrane stain into the tissue blocks. The generation of reduced Os species ( $\mathrm{Os}{ }^{\mathrm{VI}}$ and $\mathrm{Os}^{\mathrm{IV}}$ ) only via the dihydroxilation (Fig. 3a), however, may saturate with decreasing availability of unsaturated membrane lipids. Both effects could explain why the OTO protocol ${ }^{16}$ (which involves two $\mathrm{OsO}_{4}$ incubation steps and an intermittent thiocarbohydrazide $(\mathrm{TCH})$ enhancing reaction, but no reducing agent, Fig. 3c) provides good stain penetration but low contrast (limited by availability of unsaturated membrane lipids). In addition, $\mathrm{OsO}_{4}$ can be washed out in this protocol during the washing steps following $\mathrm{OsO}_{4}$ incubation (via reaction -1 ).

On the other hand, reduced Os protocols ( $\mathrm{rOTO}^{17}$; using ferrocyanide/ $/ \mathrm{OsO}_{4}$ mixture instead of $\mathrm{OsO}_{4}$, also used in recent studies $^{18,19}$, Fig. 3d) 'push' almost the entire water-dissolved Os VIII fraction into the membrane thus avoiding unsaturated lipid depletion and increasing local contrast, but hampering further membrane penetration. This can explain why the reduced Os staining protocols (Fig. 3d) provide very good contrast in the periphery, but with limited penetration (typically $<200 \mu \mathrm{m}$ from the tissue surface).

This logic motivates our alteration of the staining protocol (Fig. 3e): we first allow $\mathrm{OsO}_{4}$ to penetrate deep into the tissue (via steps $1,-1$ ) and then immediately apply the reducing agent ferrocyanide (without an intermediate washing step, which would extract Os ${ }^{\mathrm{VI}}$ or Os ${ }^{\mathrm{VIII}}$ from the membrane). 
a
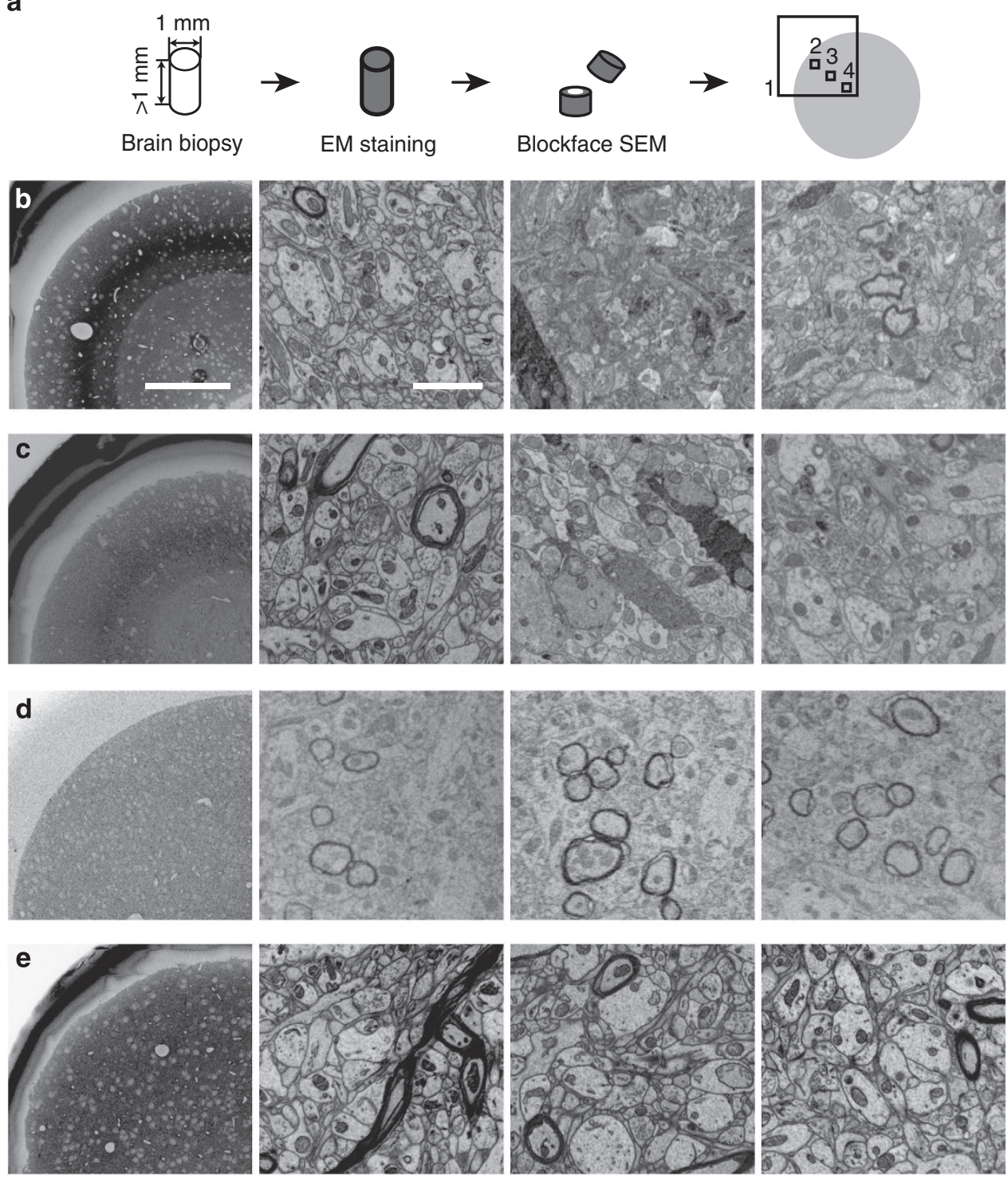

Figure 1 | Novel large-scale en-bloc EM staining protocol for dense connectomic circuit reconstruction: protocol comparison. (a) Screening strategy for brain biopsy samples $>1 \mathrm{~mm}$ in smallest dimension. Samples were screened after cutting the sample in about half, such that the core of the sample was exposed and could be tested for staining quality. Samples were screened in overview (1), periphery (2), intermediate (3) and core regions (4).

(b-e) Scanning electron microscopy images from mouse cortex biopsy samples screened as indicated in a (regions 1-4, left to right columns) and stained with en-bloc protocols from ref. 18 (b, rOTO), ref. 19 (c, rOTO) and ref. 2 (d, PATCO) and this protocol (e) Note the good staining quality in the periphery (first column) in $\mathbf{b}, \mathbf{c}$ but the strong over-staining in the intermediate region yielding stain uptake of a subset of neurons (b,c) and a substantial staining gradient, which is relieved in this protocol. (e) PATCO provides good stain penetration but stains myelinated neurites only, (d) Scale bars, $200 \mu \mathrm{m}$ in b-e left column and $2 \mu \mathrm{m}$ in other columns.

How did we arrive at this protocol alteration? Initially, we attempted to use periodic acid (which was so far used to stain myelinated fibres ${ }^{2,20}$ ), however, together with $\mathrm{OsO}_{4}$, such that periodic acid could attach to dihydroxylated lipids and make the binding site again available for a further round of Os amplification through TCH. However, the effect of periodic acid application after $\mathrm{OsO}_{4}$ incubation (Fig. 3f) was opposite: membrane staining contrast got worse (Fig. 3g). We also noted that periodic acid weakened the strong macroscopic tissue staining (black under bright-field illumination, Fig. 3f). Since the most likely source of this heavy black staining is membrane-dissolved or -precipitated $\mathrm{OsO}_{2}$ ('Os black'), we therefore wondered whether in fact periodic acid had oxidized $\mathrm{OsO}_{2}$ to water soluble and colourless $\left[\mathrm{OsO}_{4}(\mathrm{OH})_{2}\right]^{2-}$.
This made us consider an addition to the classic concept of Os membrane staining. So far, specific chemical binding of $\mathrm{OsO}_{4}$ to unsaturated lipids was considered the main source of long-term membrane staining ${ }^{11,12}$. But what if membrane-dissolved blackcolored $\mathrm{OsO}_{2}$ was a parallel and crucial source of membrane contrast? Thus the macroscopically black appearance of the $\mathrm{OsO}_{4}$-impregnated brain (Fig. 3f, middle) could result from $\mathrm{OsO}_{2}$ enriched in membranes. A simple water/octanol partition test (Fig. 3h) provided support for this hypothesis: when mixing equal parts of water-dissolved $2 \% \mathrm{OsO}_{4}$ and octanol, the organic and aqueous phases separated (Fig. 3h, left). After several hours, however, the organic phase had turned deep black (Fig. 3h, right). Since 1-octanol can progressively reduce $\mathrm{OsO}_{4}$ to $\mathrm{OsO}_{2}$, but cannot form stable osmate esters due to the absence of carbon-carbon double bonds the partition of black compounds in 

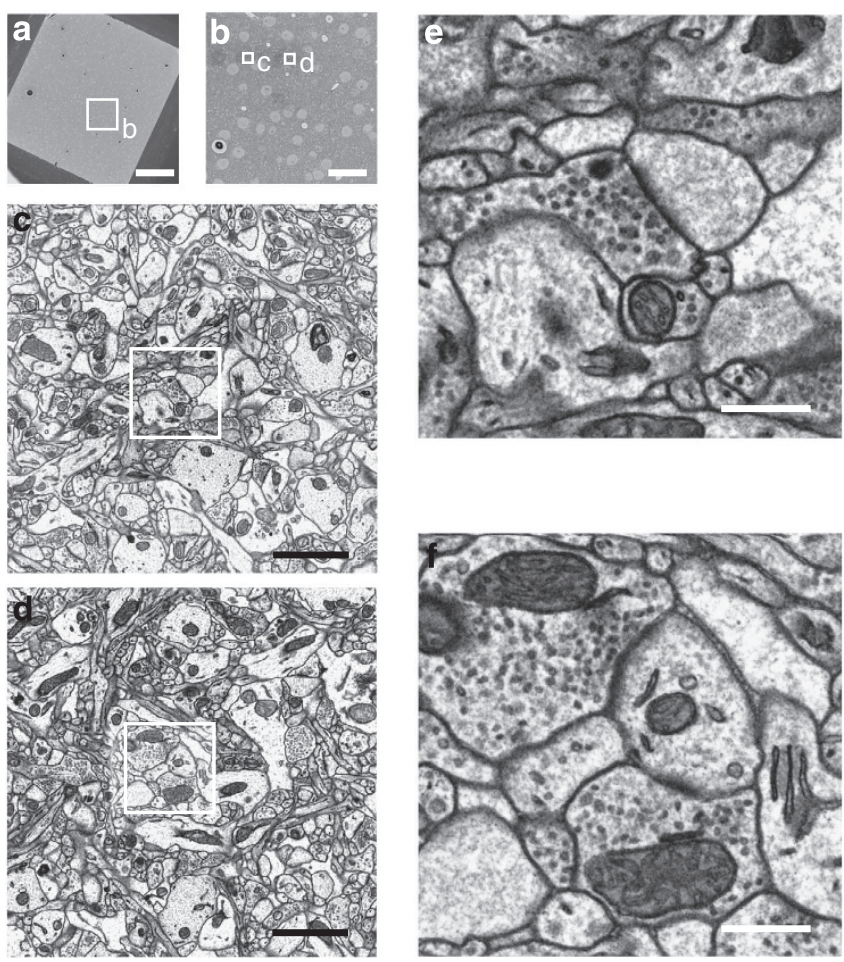

Figure 2 | High-resolution screening of ultrastructure and staining contrast at the core of $\sim 1-\mathbf{m m}$-sized tissue sample. (a-d) Position of screening region within sample. (e,f) Two example high-resolution scanning electron microscopy images (imaged at voxel size of $1.35 \times 1.35 \mathrm{~nm}$ ) showing continuous strong membrane contrast in plasma and vesicle membranes, indications of postsynaptic density, and intracellular

organelles. Images in e,f were imaged first on a freshly cut block face, and images in a-d were taken afterwards to avoid effects of multiple exposure in the high-resolution images. See Supplementary Fig. 1 for further high-

resolution screening results. Scale bars, $200 \mu \mathrm{m}$ in $\mathbf{a}, 40 \mu \mathrm{m}$ in $\mathbf{b}, 2 \mu \mathrm{m}$ in c,d and $500 \mathrm{~nm}$ in e,f

the lipophilic phase as seen in the partition test (Fig. 3h) is likely caused by the strongly lipophilic $\mathrm{OsO}_{2}$.

In summary, with respect to Os staining, our protocol follows the logic that $\mathrm{OsO}_{4}$ needs to stay in VIII-oxidized form for subsequent membrane uptake events and thus tissue penetration; but it needs to be reduced to oxidation level IV $\left(\mathrm{OsO}_{2}\right)$ to be kept in the membrane for high-contrast tissue staining.

With respect to uranium staining, we hypothesized that the temperature dependency of UA-substrate interactions was stronger for the chemical reactions leading to binding of UA to protein and membrane targets ${ }^{10}$ than for its diffusibility through tissue. We therefore exposed the samples to a two-step UA immersion: first overnight at low temperature $\left(4^{\circ} \mathrm{C}\right)$ to allow diffusion over large distances; and then a brief step to $50^{\circ} \mathrm{C}$ (for $2 \mathrm{~h}$ ) to enhance reactivity with protein and membrane targets. The latter enhanced staining contrast (Supplementary Fig. 2). In addition, we optimized the dehydration and resin-embedding steps for larger tissue volumes (see Methods).

The protocol was then controlled for inter-experimentator variability and for variability between cortical areas (four independent experimentators; somatosensory and parietal cortex of mouse, Fig. 1e, Supplementary Fig. 3, Supplementary Table 2).

We finally tested whether the new protocol in fact provides sufficient staining contrast for dense circuit reconstruction (Fig. 4). For this, we acquired a three-dimensional (3D) serial block-face electron microscopy (SBEM) image data set sized
$65 \times 51 \times 41 \mu \mathrm{m}^{3}$ with a voxel size of $12 \times 12 \times 30 \mathrm{~nm}^{3}$ (Fig. 4 b, Supplementary Movie) from the core of one 1-mm-sized sample (at about $500 \mu \mathrm{m}$ from the sample border, Fig. 4a) and asked 7 trained annotators to start at 116 random neurite seed points in the data set (see Methods for details of the sampling procedure). From each seed point, the annotators were asked to reconstruct the respective neurite within a cubic bounding box of $10 \mu \mathrm{m}$ edge length (Fig. $4 \mathrm{~b}, c$; average traced path length per seed was $27.9 \mu \mathrm{m}$ including branches), yielding a total tracing of $22.7 \mathrm{~mm}$ path length. We then applied the RESCOP skeleton consensus computation $^{21}$, which measures the distribution of agreement between tracers (vote histogram, Fig. 4d). We then used RESCOP to determine the traceability prior (Fig. $4 \mathrm{f}$ ), which reports the distribution of tracing difficulty in the data set, by fitting to the measured vote histogram (Fig. 4d reports the measured and Fig. 4e the fitted vote histogram). Finally, RESCOP provides a prediction of tracing accuracy in dependence of the number of redundant annotations (Fig. 4g). This quantification allows the comparison to tracing precision in both the published retina data sets $^{18,21,22}$ and to a data set acquired from mouse somatosensory cortex but stained with the conventional en-bloc protocol (protocol from ref. 18, see Fig. 1b; data set: Boergens et al., unpublished data set). We find that the dense reconstructions performed at the core of a sample stained with our new protocol are at least as good as those from the retina data set and a conventionally-stained cortex data set (Fig. 4g). Thus, our staining quality is comparable to the previous (volume limited) protocols and allows for dense neuronal circuit reconstruction.

\section{Discussion}

The reported EM staining protocol is currently mainly aimed at yielding consistently high-contrast staining for samples sized about a millimetre in diameter. This is an enormous alleviation of the experimental burden in EM-based connectomics. In many experimental settings, the neuronal tissue-of-interest is a structure of several hundred micrometres in diameter (for example, barrels in mouse S1 cortex, subnuclei in the thalamus or directional preference columns in visual cortex). With the existing protocols, the maximum sample size was just on that same spatial scale, that is, one had to extract samples of about $400 \mu \mathrm{m}$ in diameter at about $10-50 \mu \mathrm{m}$ targeting precision from entire mouse brains (Fig. 4h). This represented a substantial obstacle in en-bloc EM imaging. Our protocol enables sample volumes that considerably relieve the precision requirements in sample targeting (Fig. 4h).

While such obstacles may be just acceptable for purely structural investigations (where multiple samples can be readily obtained at low resource consumption), this sampling statistics was close to prohibitive for correlated functional/structural experiments. In these settings, the functional experiment alone has usually challenging success statistics (often one-fifth successful experiments). Increasing this sampling burden by a factor of about 5-10 makes such correlated experiments close to impossible. Therefore, our protocol not only makes large-scale structural imaging possible, but substantially alleviates the EM en-bloc staining challenges encountered in correlated experimental settings, opening the path to many promising structure-function studies in the emerging field of connectomics.

We tested our protocol for samples sized about $1 \mathrm{~mm}$ in diameter. In principle, an extension of the protocol to samples at the size of a hemisphere or an entire mouse brain should be possible by prolonging the respective impregnation steps. With larger samples, however, macroscopic sample damage becomes more likely, such as cracks in the tissue blocks. With highcontrast staining, another challenge is maintaining conductivity 
a

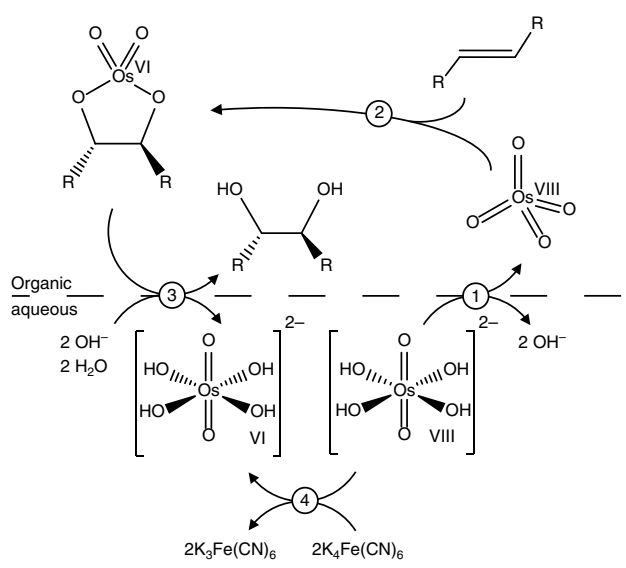

b

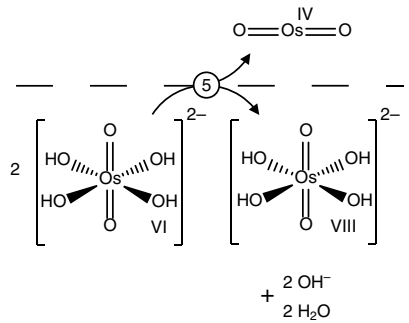

f

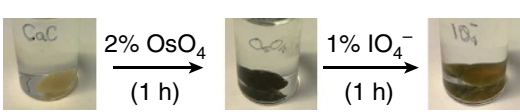

g

h
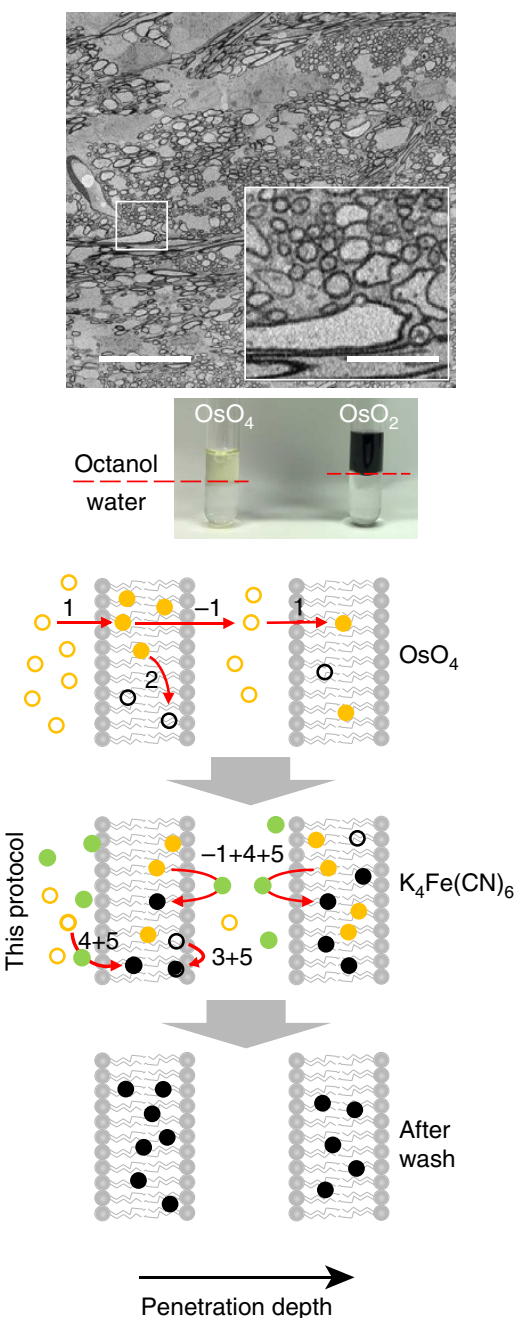

Penetration depth

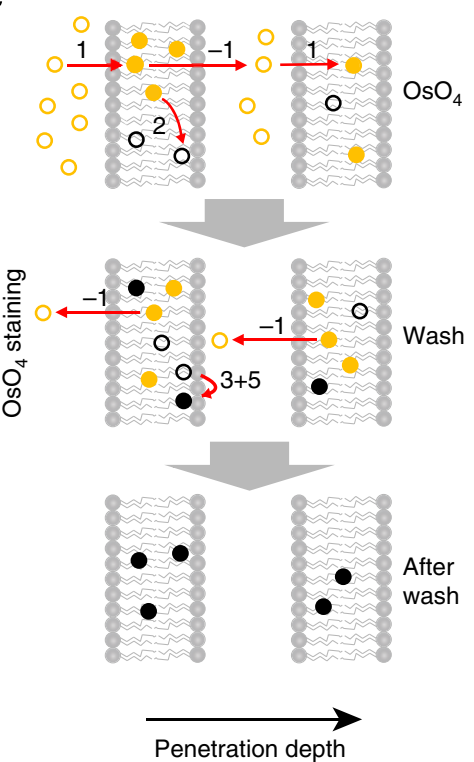

d

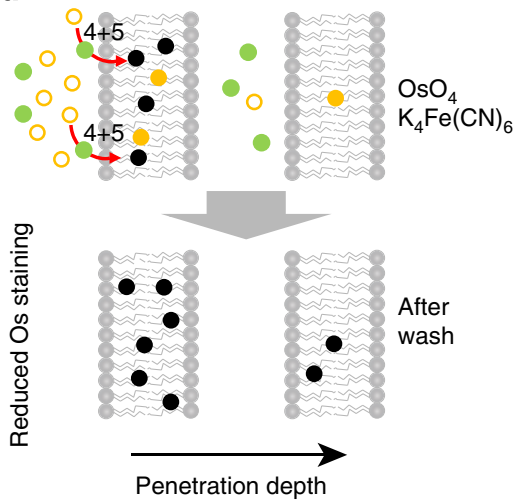

- $\mathrm{OsO}_{4}$

- $\left[\mathrm{OsO}_{4}(\mathrm{OH})_{2}\right]^{2-}$

- $\mathrm{K}_{4} \mathrm{Fe}(\mathrm{CN})_{6}$

- $\mathrm{OsO}_{3}-\mathrm{R}$

- $\mathrm{OsO}_{2}$

Figure 3 | Possible chemical reactions underlying Os-based membrane staining and logic of our staining protocol. (a) Sharpless-dihydroxilation (modified from ref. 9) and possible effect of reducing agents such as ferrocyanide. Note that reaction steps 1-5 are referred to in Results, and inverse reaction steps are referred to as ' -1 ' to ' $-5^{\prime}$. (b) Os ${ }^{I V}$ dismutation suggested to be critical for deposition of $\mathrm{OsO}_{2}$ in the membrane (after ref. 12) (c-e) Possible reaction and diffusion steps involved in stain penetration and contrast enhancement in the conventional OTO protocol, (c) rOTO protocol (d, see also Fig. 1b,c) and this protocol (e, compare with Fig. 1e). Two membranes are visualized to indicate penetration of successive plasma and intracellular membrane bilayers. Only key reaction steps are indicated. (f) Simple test experiment that lead us to consider $\mathrm{OsO}_{2}$ as a main source of membrane contrast. After Os impregnation, the tissue obtains a dark black colour under bright-field illumination (middle). This is reversed by periodic acid application (right), suggesting that $\mathrm{OsO}_{2}$ is oxidized to colourless Os species. (g) EM contrast after periodic acid application was decreased, staining only myelinated fibres (inset, note region between myelinated fibres has very low contrast making the detection of single neurites impossible), suggesting that $\mathrm{OsO}_{2}$ had been essential for Os membrane contrast. (h) Simple octanol/water partition test: equal fractions of OsO $\mathrm{O}_{4}$ in aqueous solution and octanol partition into organic (top) and aqueous (bottom) phase. After several hours (right) the lipophilic phase is deep black, supporting the notion that Os $\mathrm{O}_{2}$ (which is the product of $\mathrm{OsO}_{4}$ reduction by octanol) can be dissolved in lipophilic membranes and may contribute crucially to membrane contrast. Scale bars, $20 \mu \mathrm{m}$ and $5 \mu \mathrm{m}$ in $\mathbf{g}$ and insert, respectively.

throughout large sample volumes, since the less metal deposition in the cytosol (less 'background') the less conductive the sample. Using conductive resins or increasing cytosol staining may relieve these challenges for very large samples.

Astonishingly, our protocol abolishes a decade-long obstacle to EM staining with only a few targeted modifications of en-bloc staining. These were, however, based on particular models of the deposition of Os in biological membranes. At the core is the notion that $\mathrm{OsO}_{2}$ substantially contributes to membrane contrast. This is far from proven, and further experiments may elucidate the contributions of Os at low oxidation states to membrane contrast in biological samples. While only tested for neuropil, our protocol may be well-applicable to other forms of tissue, where $3 \mathrm{D}$ EM reconstruction is desirable.

\section{Methods}

Animals. Male mice (P28-P30) were anesthetized and killed by transcardial perfusion of fixative. All procedures were approved by the local animal care and use committee and were in accordance with the laws of animal experimentation issued by the German federal government. 
a

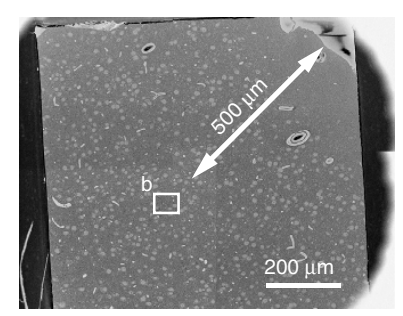

d

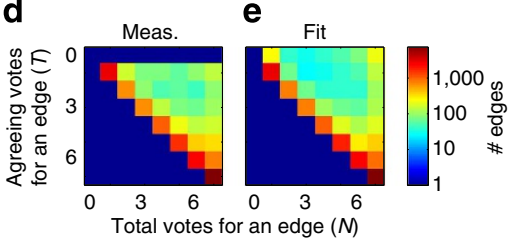

f

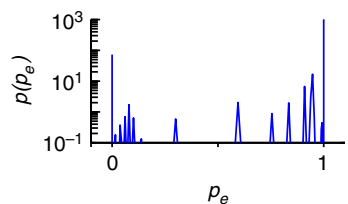

b

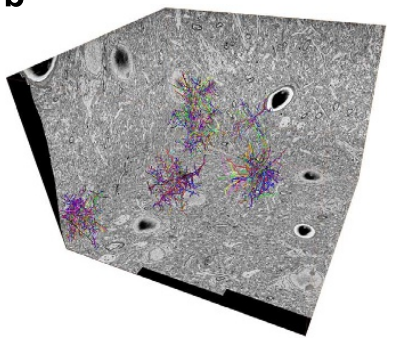

$\mathbf{g}$

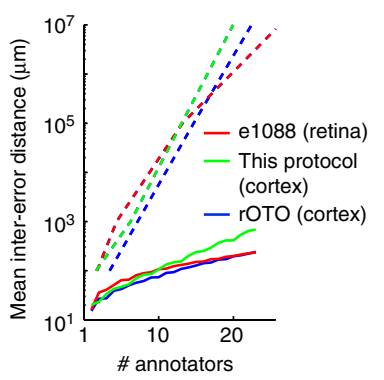

C

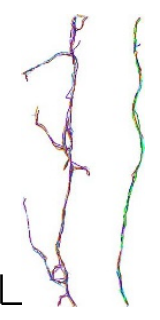

h

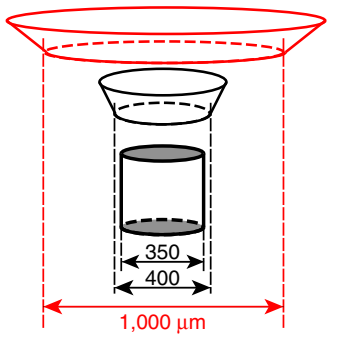

Figure 4 | Dense neurite reconstruction in large tissue blocks. (a,b) Dense neurite reconstruction in a SBEM data set sized $65 \times 51 \times 41 \mu \mathrm{m}^{3}$ from the core $(>500 \mu \mathrm{m}$ from the closest sample surface) of a sample from mouse somatosensory cortex. (a) Overview of sample location (stitched from four overview images); (b) 3D display of SBEM-data set boundaries and all locally dense neurite tracings used for tracing test. (c) Two example neurites reconstructed by seven independent annotators. Note complete agreement (right) and local disagreement when tracing spines (left).

$(\mathbf{d}, \mathbf{e})$ Measured $(\mathbf{d})$ and fitted $(\mathbf{e})$ vote histogram of tracer agreement reporting the total number of tracers $(N)$ and the agreeing number of tracers $(T)$ for each skeleton-edge (redundancy-corrected, see Methods). (f) fitted distribution of skeleton-edge difficulty (or edge probability) $p\left(p_{e}\right)$ from the tracings summarized in d, yielding the fitted vote histogram (e, see RESCOP ${ }^{21}$ and Methods for details). (g) Prediction of tracing accuracy (for full neurite tracings, continuous lines and for focused re-annotation of the disagreeing locations, dashed lines, see ref. 21) from test tracings for this data set compared with published retina tracings ('e1088' from ref. 21) and a comparison cortex data set stained with the protocol as shown in Fig. 1b (Boergens et al., unpublished data set). (h) Illustration of sampling challenge when targeting modules in cortex (here: 'barrels' in mouse S1 layer 4, grey cylinder) using a protocol that can only stain about 400- $\mu \mathrm{m}$-wide samples (black) compared with the procedural relief when obtaining about 1-mm-wide samples (red). Scale bars, $200 \mu \mathrm{m}(\mathbf{a}), 1 \mu \mathrm{m}(\mathbf{c})$.

Sample extraction and fixation. Animals were anesthetized with isoflurane (Baxter) inhalation and perfused with $15 \mathrm{ml}$ cacodylate (Serva, Heidelberg, Germany) buffer ( $0.15 \mathrm{M}, \mathrm{pH} 7.4)$ followed by $30 \mathrm{ml}$ fixative mixture containing $0.08 \mathrm{M}$ cacodylate ( $\mathrm{pH} 7.4$ ), 2.5\% paraformaldehyde (Sigma-Aldrich, St Louis, USA), $1.25 \%$ glutaraldehyde (Serva) and $2 \mathrm{mM}$ calcium chloride (Sigma-Aldrich). The brain was removed from the skull with care to avoid mechanical irritation and post-fixed for 12 to $24 \mathrm{~h}$ at $4{ }^{\circ} \mathrm{C}$ in the fixative mixture. Then a biopsy punch (KAI Medical, Honolulu, USA) was used to extract samples sized about $1 \mathrm{~mm}$ in diameter and about $2 \mathrm{~mm}$ in length.

Sample staining. Large-volume en-bloc staining was performed as follows (also see Supplementary Table 1 for details). Tissue was first immersed in $2 \% \mathrm{OsO}_{4}$ aqueous solution (Serva) buffered with cacodylate $(0.15 \mathrm{M}, \mathrm{pH} 7.4)$ at room temperature for $90 \mathrm{~min}$. The staining buffer was then replaced by $2.5 \%$ ferrocyanide (Sigma-Aldrich) in $0.15 \mathrm{M}$ cacodylate buffer (pH 7.4) and incubated at room temperature for another $90 \mathrm{~min}$. Sequentially, the tissue was incubated in filtered thiocarbohydrazide (saturated aqueous solution at room temperature, SigmaAldrich) at $40{ }^{\circ} \mathrm{C}$ for $45 \mathrm{~min}, 2 \%$ unbuffered $\mathrm{OsO}_{4}$ aqueous solution at room temperature for $90 \mathrm{~min}$ and $1 \% \mathrm{UA}$ (Serva) aqueous solution at $4{ }^{\circ} \mathrm{C}$ overnight. Double rinses in nanopure filtered water for $30 \mathrm{~min}$ each were performed between the ferrocyanide and thiocarbohydrazide step, the thiocarbohydrazide and $\mathrm{OsO}_{4}$ step, and the $\mathrm{OsO}_{4}$ and UA step. On the next day, the tissue (still in UA solution) was warmed up to $50^{\circ} \mathrm{C}$ (oven) for $120 \mathrm{~min}$. After being washed twice in nanopure filtered water at room temperature for $30 \mathrm{~min}$, the tissue was incubated in a lead aspartate solution at $50^{\circ} \mathrm{C}$ for $120 \mathrm{~min}$. The lead aspartate solution was prepared by dissolving $0.066 \mathrm{~g}$ lead nitrate (Sigma-Aldrich) in $10 \mathrm{ml} 0.03 \mathrm{M}$ aspartic acid (Serva) and $\mathrm{pH}$ adjusted to 5 with $1 \mathrm{~N} \mathrm{KOH}$. The tissue was then washed twice in nanopure filtered water for $30 \mathrm{~min}$. The image in Supplementary Fig. 2b was taken from a tissue block stained with the same procedure described above except that the 120 min $50^{\circ} \mathrm{C}$ incubation in UA was omitted.

Sample embedding. For embedding, samples were dehydrated through a graded ethanol series $\left(50,75,100 \%, 30 \mathrm{~min}\right.$ each, all cooled at $\left.4^{\circ} \mathrm{C}\right)$ into pure acetone
( $3 \times 100 \%, 30 \mathrm{~min}$ at room temperature) followed by epoxy-monomer infiltration by immersion into 1:1 mixtures of acetone and Spurr's resin (4.1 g ERL 4221, $0.95 \mathrm{~g}$ DER 736 and $5.9 \mathrm{~g} \mathrm{NSA}$; Sigma-Aldrich) at room temperature for $12 \mathrm{~h}$ or overnight on a rotator (samples were maintained in an open Eppendorf tube). Infiltrated samples were then incubated in pure resin with 1\% DMAE for $6 \mathrm{~h}$ (in closed Eppendorf tubes) and placed in embedding moulds (Polyscience, Eppelheim, Germany) in a pre-warmed oven $\left(70^{\circ} \mathrm{C}\right)$ for 48 to $72 \mathrm{~h}$.

Sample screening. Embedded samples were trimmed to a block face of $1.5 \times 1.5 \mathrm{~mm}^{2}$ and imaged in a scanning electron microscope with a field-emission cathode (QuantaFEG 200, FEI Company). An incident electron beam with a spot size of 2.9 and an energy of $2.8 \mathrm{keV}$ was used to scan across the samples at a pixel dwell time of $3.2 \mu$ s and a pixel size of $6.1 \times 6.1 \mathrm{~nm}$ (Fig. $1 \mathrm{~b}$,c,e and Supplementary Fig. 2) or $12 \times 12 \mathrm{~nm}$ (Fig. 1d). Electrons were detected using a custom-designed detector based on a silicon diode (AXUV, International Radiation Detectors) combined with a custom-built current amplifier (courtesy of W. Denk, MPI Heidelberg), as described previously ${ }^{18,22}$. To prevent charging for PATCOstained samples, the chamber was kept at a pressure of $0.20 \mathrm{mbar}$, while other samples were imaged at high vacuum. All samples were either mounted onto aluminium holders with electrical conductive glue (Henkel, Düsseldorf, Germany) or connected with conductive silver paint (SPI suppliers, West Chester, USA) to the metal stage.

High-resolution images (Fig. 2 and Supplementary Fig. 1) were taken from a tissue block mounted on an aluminium pin with electrically conductive glue. The tissue block was then trimmed at about $500-\mu \mathrm{m}$ depth from the pia and at about $100 \mu \mathrm{m}$ from each other side, resulting in a block face with a size of about $970 \times 890 \mu \mathrm{m}$. The sample was then coated with a gold layer of 200-nm thickness, the block face was exposed again by carefully trimming off the surface with an ultra-microtome (Leica, UC7). EM micrographs were taken in a scanning electron microscope (Verios 460, FEI company) in high vacuum mode. An incident electron beam with an energy of $2.8 \mathrm{keV}$ and a current of $0.2 \mathrm{nA}$ was applied to scan across the sample at a pixel dwell time of $10 \mu$ s with a pixel size of $1.35 \times 1.35 \mathrm{~nm}$ or $5.62 \times 5.62 \mathrm{~nm}$. 
SBEM stack acquisition. To test tissue cuttability and for performing tracing test experiments, we acquired a 3D SBEM data set $(3 \times 3$ image tiles; single image size $2,048 \times 1,768$ pixels; lateral overlap between image tiles about $9 \%$ in $x$-direction and $6 \%$ in $y$-direction, respectively; 1,370 slices at nominal cutting thickness of $30 \mathrm{~nm}$; incident beam energy $2.8 \mathrm{keV}$; dwell time $3.2 \mu \mathrm{s}$; data set size $65 \times$ $51 \times 41 \mu \mathrm{m}^{3}$; voxel size $12 \times 12 \times 30 \mathrm{~nm}^{3}$ ) from one of the samples processed with our protocol (Supplementary Table 1, Fig. 4a)

Neurite reconstruction and traceability computations. To generate tracing seed points, six image regions of size $2 \times 2 \mu \mathrm{m}$ each were randomly selected from the data set. In these regions, all cross-sections of neuronal or glial processes were manually marked. Then, glial processes and cell bodies were excluded, leaving a total of 116 neurite processes. These were delivered to annotators as starting (seed) points. Annotators were asked to trace the respective neurite in all directions starting at the respective seed point, within a cubic bounding box of $10 \mu \mathrm{m}$ edge length, centred on the seed point. This yielded $116 \times 7=812$ single skeleton tracings, $22.7 \mathrm{~mm}$ total path length. These were then analysed using the redundant skeleton consolidation procedure ( RESCOP $^{21}$ ).

Briefly, each set of seven redundant skeletons was compared computing the number of 'pro' votes $(T)$ and total votes $(N)$ for each edge in each skeleton tracing (parameters: 'initial correspondence test radius' $r_{\mathrm{p}}$ : $225 \mathrm{~nm}$; r.m.s. node distance threshold $\Theta=375 \mathrm{~nm}$, see code in Supplementary Software). The resulting vote histogram was corrected for the redundancy $(N)$ of each tracing, yielding the measured vote histogram (Fig. $4 \mathrm{~d}$ ). Then, the underlying prior of edge probability $p\left(p_{e}\right)$ was determined by fitting the vote histogram under the simplifying assumption that tracing decisions were independent between tracers and locations (see ref. 21 for details). The best-fit prior and resulting predicted vote histogram are shown in Fig. 4e,f. Then, the predicted mean error-free path length as a function of the number of tracings per seed point ('tracing redundancy') was computed from the fitted prior $p\left(p_{e}\right)$ (solid lines in Fig. $4 \mathrm{~g}$ ) for the setting of annotators reconstructing an entire neuron/neurite; and for the setting of focused annotation where skeleton edges are repeatedly annotated until a set accuracy goal is reached (dashed lines in Fig. 4g, see ref. 21 for details). The code for this analysis (MATLAB, Mathworks) can be found in the Supplementary Software together with all tracings. For comparison, the published retina data ${ }^{21}$ was re-analysed based on the measured vote histogram using the same code, as was the comparison data set from rOTO-stained mouse cortex (Boergens et al., unpublished data set).

Exploratory test experiments. To ensure that the new protocol is readily applicable to other cortical areas and can be easily implemented by other experimentalists, we asked two students in the laboratory to use the protocol without direct supervision (just based on written instructions). Both were able to conduct successful stainings in somatosensory and parietal cortex (Supplementary Fig. 3 and Supplementary Table 2), though one sample showed charging in parts of the volume (Supplementary Fig. 3c). Successful protocol applications were required between 1 and about 10 training iterations for experienced and novice students, respectively.

\section{References}

1. Briggman, K. L. \& Bock, D. D. Volume electron microscopy for neuronal circuit reconstruction. Curr. Opin. Neurobiol. 22, 154-161 (2012).

2. Mikula, S. \& Denk, W. High-resolution whole-brain staining for electron microscopic circuit reconstruction. Nat. Methods 12, 541-546 (2015).

3. Helmstaedter, M. Cellular-resolution connectomics: challenges of dense neural circuit reconstruction. Nat. Methods 10, 501-507 (2013).

4. Palade, G. E. A study of fixation for electron microscopy. J. Exp. Med. 95, 285-298 (1952).

5. Watson, M. L. Staining of tissue sections for electron microscopy with heavy metals. J. Biophys. Biochem. Cytol. 4, 475-478 (1958).

6. Tapia, J. C. et al. High-contrast en bloc staining of neuronal tissue for field emission scanning electron microscopy. Nat. Protoc. 7, 193-206 (2012).

7. Wai, J. S. M. et al. A mechanistic insight leads to a greatly improved osmium-catalyzed asymmetric dihydroxylation process. J. Am. Chem. Soc. 111, $1123-1125$ (1989).

8. Kwong, H.-L., Sorato, C., Ogino, Y., Chen, H. \& Sharpless, K. B. Preclusion of the "second cycle" in the osmium-catalyzed asymmetric dihydroxylation of olefins leads to a superior process. Tetrahedron Lett. 31, 2999-3002 (1990).

9. Kolb, H. C., VanNieuwenhze, M. S. \& Sharpless, K. B. Catalytic Asymmetric Dihydroxylation. Chem. Rev. 94, 2483-2547 (1994).

10. Locke, M., Krishnan, N. \& McMahon, J. T. A routine method for obtaining high contrast without staining sections. J. Cell Biol. 50, 540-544 (1971).
11. Khan, A. A., Riemersma, J. C. \& Booij, H. L. The reactions of osmium tetroxide with lipids and other compounds. J. Histochem. Cytochem. 9, 560-563 (1961).

12. Korn, E. D. A chromatographic and spectrophotometric study of the products of the reaction of osmium tetroxide with unsaturated lipids. J. Cell Biol. 34 627-638 (1967).

13. White, D. L., Andrews, S. B., Faller, J. W. \& Barrnett, R. J. The chemical nature of osmium tetroxide fixation and staining of membranes by $\mathrm{x}$-ray photoelectron spectroscopy. Biochim. Biophys. Acta 436, 577-592 (1976).

14. Subbaraman, L. R., Subbaraman, J. \& Behrman, E. J. Inorganic Chemistry (Department of Biochemistry, Ohio State Univ.11, 2621-2627, 1972).

15. White, D. L., Mazurkiewicz, J. E. \& Barrnett, R. J. A chemical mechanism for tissue staining by osmium tetroxide-ferrocyanide mixtures. J. Histochem. Cytochem. 27, 1084-1091 (1979).

16. Seligman, A. M., Wasserkrug, H. L. \& Hanker, J. S. A new staining method (OTO) for enhancing contrast of lipid--containing membranes and droplets in osmium tetroxide--fixed tissue with osmiophilic thiocarbohydrazide(TCH). J. Cell Biol. 30, 424-432 (1966).

17. Willingham, M. C. \& Rutherford, A. V. The use of osmiumthiocarbohydrazide-osmium (OTO) and ferrocyanide-reduced osmium methods to enhance membrane contrast and preservation in cultured cells. J. Histochem. Cytochem. 32, 455-460 (1984).

18. Briggman, K. L., Helmstaedter, M. \& Denk, W. Wiring specificity in the direction-selectivity circuit of the retina. Nature 471, 183-188 (2011).

19. Holcomb, P. S. et al. Synaptic inputs compete during rapid formation of the calyx of Held: a new model system for neural development. J. Neurosci. 33, 12954-12969 (2013).

20. Seligman, A. M., Hanker, J. S., Wasserkrug, H., Dmochowski, H. \& Katzoff, L. Histochemical demonstration of some oxidized macromolecules with thiocarbohydrazide (tch) or thiosemicarbazide (TSC) and osmium tetroxide. J. Histochem. Cytochem. 13, 629-639 (1965).

21. Helmstaedter, M., Briggman, K. L. \& Denk, W. High-accuracy neurite reconstruction for high-throughput neuroanatomy. Nat. Neurosci. 14, 1081-1088 (2011).

22. Helmstaedter, M. et al. Connectomic reconstruction of the inner plexiform layer in the mouse retina. Nature 500, 168-174 (2013).

\section{Acknowledgements}

We thank Kevin Briggman, Winfried Denk, Mark Ellisman, Kristen Harris and Shawn Mikula for fruitful discussions; Ali Karimi, Florian Drawitsch, Jakob Straehle and Yates Buckley for comments on the manuscript; Ali Karimi and Anjali Gour for performing protocol test experiments; Heiko Wissler for excellent help with tracer management and figure generation; Vera Nijveld for support with schematics; and Lisa Geiser, Raphael Jakoby, Raphael Kneissl, Vanessa Robl, Nathalie Schmidt and Victoria Schuhbeck for neurite reconstructions.

\section{Author contributions}

Y.H. developed the protocol and carried out the experiments. P.L. contributed to protocol optimization and carried out experiments. Y.H. and M.H. analysed the data. Y.H. and M.H. wrote the paper.

\section{Additional information}

Supplementary Information accompanies this paper at http://www.nature.com/ naturecommunications

Competing financial interests: The authors declare no competing financial interests

Reprints and permission information is available online at http://npg.nature.com/ reprintsandpermissions/

How to cite this article: Hua, Y. et al. Large-volume en-bloc staining for electron microscopy-based connectomics. Nat. Commun. 6:7923 doi: 10.1038/ncomms8923 (2015)

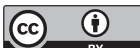

This work is licensed under a Creative Commons Attribution 4.0 International License. The images or other third party material in this article are included in the article's Creative Commons license, unless indicated otherwise in the credit line; if the material is not included under the Creative Commons license, users will need to obtain permission from the license holder to reproduce the material. To view a copy of this license, visit http://creativecommons.org/licenses/by/4.0/ 